\title{
Childhood and Adolescence Between Past and Present. Using Knowledge Organization to Bridge the Different Channels of a Cultural Institution: The Case of the Istituto degli Innocenti, Firenze
}

\author{
Luca Rosati*, Antonella Schena**, and Rita Massacesi*** \\ * University for Foreigners of Perugia, Piazza Fortebraccio 4, 06123 Perugia - Italy, \\ $<$ luca@1ucarosati.it> \\ ** Istituto degli Innocenti, Piazza SS. Annunziata, 12 - 50122 Florence (Italy), \\ <schena@istitutodeglinnocenti.it> \\ *** Istituto degli Innocenti, Piazza SS. Annunziata, 12 - 50122 Florence (Italy), \\ $<$ massacesi@istitutodeglinnocenti.it>
}

\begin{abstract}
Luca Rosati is an independent information architect and adjunct professor in Information Architecture at the University for Foreigners in Perugia (Italy). He supports an enlarged vision of knowledge organization-i.e., its application to every shared information environment (physical, digital, mixed) in order to achieve a unified model of human-information interaction across all the media and contexts. His latest book, Pervasive Information Architecture: Designing Cross-Channel User Experiences (Morgan Kaufmann 2011, with Andrea Resmini), has been dedicated to this idea. His website is http://lucarosati.it.

Antonella Schena works at Istituto degli Innocenti in the field of documentation on children's rights. She is the director of both the Innocenti Library A.C. Moro, a joint cooperation project with UNICEF Research Office according to an agreement with the Italian Government, and of the historical archive. She coordinated the activity for the construction of the Thesaurus on Childhood and Adolescence, published in 2007 (available on http://www.minori.it/thesaurus), which is used for the indexing and searching by subject of documents in the OPAC(http://opac.minori.it/lexicon/opac.aspx). She also coordinates the implementation of the new website of the National Childhood and Adolescence Documentation and Analysis Centre of the Italian Government www.minori.it.
\end{abstract}

Rita Massacesi is an information professional. She is especially interested in subject indexing and in the development of indexing languages. She works at Istituto degli Innocenti in Florence (Italy); in particular, she supports the development of the Thesaurus (Thesaurus Italiano Infanzia e Adolescenza) and of the classification system (Schema di classificazione infanzia e adolescenza). She specialises in the application of indexing systems based on facet analysis for information retrieval. She collaborated on a specific application for for the website of the National Childhood and Adolescence Documentation and Analysis Centre.

Rosati, Luca, Schena, Antonella, and Massacesi, Rita. Childhood and Adolescence Between Past and Present. Using Knowledge Organization to Bridge the Different Channels of a Cultural Institution: The Case of the Istituto degli Innocenti, Firenze. Knowledge Organization. 40(3), 197-204. 11 references.

ABSTRACT: The paper illustrates the way a cultural institution can test a work method based on a systemic and cross-channel approach to link together the different entities and sources of knowledge on childhood and adolescence which it (the documentation centre, the historical archive, the library, the museum) owns. These channels are related one to another to create a unique ecosystem, so as to allow a seamless experience among various contexts and a systemic integration of the institution's holdings. The relationship between different channels is brought about through the use of a thesaurus; navigation between the terms in the thesaurus al-
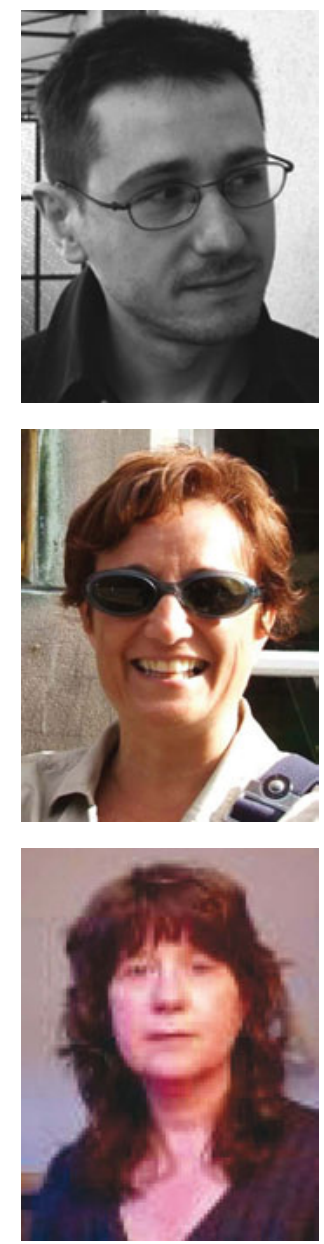

lows the creation of a consistent structure of relationships between the channels. Using the thesaurus, it is possible: to move from the digital channels of the Istituto degli Innocenti, Firenze (website, OPAC, digital repository) to the physical ones (library, museum, or ar- 
chive) using specific subjects as a compass; to pursue the visit experience by choosing specific paths within the physical space or to move from the physical to the digital world; and to develop an integrated use of web resources during a visit in physical space in order to personalise the path and find objects or related information.

Received 31 January 2013; Accepted 31 January 2013

\subsection{Introduction}

The experiment described in this article arises from the desire to create and disseminate a multifaceted, integrated knowledge of childhood as a condition, either referring to our contemporary society or in a historical perspective. This desire comes from the nature and specific mandate of the Istituto degli Innocenti, which has been carrying out activities relating to the protection of children's rights for centuries.

Founded in Florence in the first half of the fifteenth century as a place for the assistance and care of abandoned children, the Istituto degli Innocenti is now a service centre with diversified activities - it manages educational and care services for children and mothers in need; it carries out functions relating to documentation, research, and training for the Italian government (www.minori.it) and for the Regione Toscana (www.minori.toscana.it); and it holds a precious historical archive, a library (Innocenti Library A.C. Moro), and a significant artistic heritage, which will become more accessible to the public through the new museum (MUDI).

In order to link these different entities and knowledge sources on childhood and adolescence, which developed separately over a long period of time, the Istituto degli Innocenti has tested a working method based on a systemic and cross-channel approach. The aim was to initiate a transition from a silos logic (or at least a juxtapositional one) to an ecosystem one, where the whole could be bigger than the sum of its parts. The relationship between these different channels was created on a semantic basis through the use of a controlled vocabulary, the Childhood and Adolescence Italian Thesaurus (ThIA 2007); navigation between the terms in the thesaurus, linked to the individual resources, allowed the creation of a consistent and repeatable structure of relationships between them, able to guide users in their research.

At a theoretical level, an important reference point in the fulfilment of the work was either a reflection on criteria, methods, and tools, all of them necessary for the description and representation of entities belonging to different cultural areas, or the results of a long debate on the cultural web, the quality of web applications, and the interaction among users; this debate has resulted in the creation of operational tools and guidelines in Italy as well.

\subsection{Towards a common ground of Knowledge about Childhood and Adolescence}

\subsection{Background}

In Italy, as in other countries, the differences between the various kinds of cultural institutions (libraries, archives, and museums) are beginning to disappear, overcoming, in this way, limits due to the fragmentation of the cultural heritage sector. As a result, it has been necessary to reconsider the procedures and methods used in intellectual work and for the spreading of knowledge, also considering the fact that these processes increasingly take place in a digital dimension and in a web arena. There is a great deal of debate on the development of new methods and tools for the creation of integrated and straightforward access to data coming from different areas; one of the most debated questions is, on one hand, the need to safeguard the identity of the individual areas of production of this data, which reference different standards and rules for description, and on the other, to keep in mind the real needs of the various user groups to whom alternative methods of data collection to the traditional ones have been proposed (Giannetto 2011). In this context, we believe that the sharing of knowledge coming from different descriptive systems could be usefully accomplished by starting from a common semantic ground, which could be discovered in each entity regardless of its originating field, and, therefore, through index entries that are standardized and framed in a controlled vocabulary, that is precisely ThIA.

\subsection{The thesaurus (ThIA): History}

The ThIA was created between 1999-2007, through a collaboration between the National Childhood and Adolescence Documentation and Analysis Centre and the Regione Toscana. Prior to ThIA, a thesaurus specifically covering childhood didn't exist in Italy (and maybe not even abroad). The reason for that lies in the consideration that only after the approval of the 1989 UN Convention on the Rights of the Child were childhood and adolescence raised to a special significance, either from a conceptual theoretic point of view or from a political and institutional one. As a matter of fact, in the 1989 convention, a more realistic and current view of the child is stated, acknowledging him as a 
human being with his own originality, potentiality, and distinctiveness, an individual able to take active part in social life. Childhood and adolescence have attained their own specificity in the different knowledge fields, becoming a separate area of analysis, no longer necessarily related to the world of adults and parents. Up to the beginning of the ' 80 s, the social sciences, unlike disciplines such as psychology or pedagogy, showed insufficient interest towards children. They were considered a sort of appendix of someone else, and they were interesting only for what they would become ("social becoming" and not "social being"). In opposition to this traditional view of the child is the birth of a new child sociology, childhood studies, that considers children as active and qualified social actors. Another relevant contribution to increasing the value of children in their specificity was given by constructivist and interpretative theory in psychology, that has given them an active role in the developmental process, considered not only as a natural and biological fact, but also as affected by social and cultural aspects. Finally, this new vision has influenced also in a relevant way the development of children's rights, as the underage subject is not simply a receiver of protection and safeguarding on the part of adults; on the contrary, he is considered as the bearer of specific rights, including an active participation in any choices that involve him.

Moreover, the 1989 UN Convention, imposing on states that accept it the completion of real political measures that will be monitored through specific knowledge and information tools, has helped to create growing institutional and cultural attention towards children. And so, in Italy there has also been growing attention towards childhood and adolescence as a condition, attention witnessed both by the intense production of research and reports as well as by the creation of the Childhood $\mathrm{Na}$ tional Observatory, which sees in the National Childhood and Adolescence Documentation and Analysis Centre a tool in support of information and analysis. These elements determined the constitution of a specific research area and the identification of a clearly defined conceptual field (childhood and adolescence) in accordance with which it is possible to build specific semantic structures and select precise terminology: essential requirements for the creation of a specialised thesaurus.

\subsection{The thesaurus (ThLA): Main Features}

The ThIA was built taking as its main reference the standard ISO 2788-1986 for the building and development of monolingual thesauri. The vocabulary was developed starting with the selection and collection of terminology used to index documents (articles, periodicals, monographs, grey literature, audiovisual material) by the $\mathrm{Na}$ - tional and Regional Documentation Centre based on an inductive, bottom-up methodology. The thesaurus in the 2007 print version was made up of 2,953 preferred terms; it is constantly enriched and updated. In the online version, in addition to the display of the whole hierarchical structure and the navigation of relationships, it was also possible to create an interface with the catalogue of the documentation centres and permit navigation between the thesaurus terms, the subjects strings and the linked documents.

From a structural point of view, the main features of the ThIA are:

- the building methodology that follows a mixed method (organisation in either thematic areas or facets) — this method is permitted by norm ISO 2788, 9.3.2.

- polyhierarchy, that is, the possibility for some terms to have links to more than one broader term.

Therefore, the vocabulary is divided into two levels:

1. the first level, consisting of 7 thematic areas (Culture, Education, Childhood and Adolescence, Institutions, Psychological processes, Health, Society) fixes the systemic structure;

2. the second level, the faceted one, fixes the classification structure of the individual terms within the areas.

The use of polyhierarchy is strictly linked to this organisation. As a matter of fact, the decision to organise the terminology into thematic areas allowed topics concerning childhood and adolescence to be put inside a specific multidisciplinary area (childhood and adolescence), so that terms linked to this particular conceptual field are located within a specific and extended semantic network.

Alongside the main area, other different structures were developed, within which the terms linked to childhood and adolescence are located within the specific hierarchies of the different classification structures. As a matter of fact, even though polyhierarchy relates mainly to the childhood and adolescence area, it can also affect other areas.

To build structures within the thematic areas, a categorical analysis of the terms was used in order to indicate, for each term, the main category to which it belongs (e.g., actions, actors, things), and, within it, the division features, that is, the facets. The identification of the main macro-categories was also essential for verifying the terms. As a matter of fact, the terms selected for the vocabulary were submitted to a rigorous analysis in order to obtain homogeneity and univocity in the language from a formal and semantic point of view so that each term can express one and only one idea, and an idea is expressed 
always by one and only one term, getting rid of the ambiguities of natural language.

In this process, the constant reference was also the Guida all'indicizzazione per soggetto of GRIS - the research group on subject indexing of the Italian Libraries Association-in relation to the interaction between the semantic and the syntactic parts of the indexing language and its implications for the building of the vocabulary (AIB 1996).

\subsection{Applications}

\subsection{The website (mww.minori.it)}

\subsubsection{Strategy}

After the creation of the thesaurus, over a decade's worth of development in documentation and research activities for the National Childhood and Adolescence Italian Documentation and Analysis Centre required a redesign of its website. The vast amount of material in the site, organized without an appropriate and effective system for content management, revealed many problems relating to findability. Instead of starting from the beginning and using for the website a totally new and independent information architecture, the decision was made to use the thesaurus produced in the documentation area, obviously with some modifications due to the difference in medium and interface. This choice not only allowed a better correlation among the inner website resources (inner correlation), but also a strong bond between the online environment and the off-line documentation centre activities and documents (outer correlation).

The re-design of the new documentation centre website consisted of:

- inventorying and re-indexing the content

- planning new management and data search for better findability.

For information retrieval on the website, the decision was to use an indexing system based on facet analysis, created by an adaptation of the thesaurus. The use of the facet concept, introduced by Ranganathan (1965) for the development of Colon Classification, has found application also in information architecture, particularly in planning and managing websites and search engines, as it allows:

- multidimensional and flexible access to information: every facet represents a characteristic of the object, so the search can be carried out by either following a single characteristic/facet, or by putting multiple characteristics together;
- a reduction of the impact of possible changes in the search instructions as the facets represent essential and constant characteristics (persistence); and

- the possibility of adding new descriptive characteristics (facets).

The Ranganathan system also contains other essential aspects, usually little considered in web applications, such as facet citation order and a notation system useful in ensuring this order. In Italy, it has inspired studies on subject indexing which are included in the previously cited GRIS guidelines and also used by important Italian national agencies (Bianchini 2012). The decision to use the ThIA to do the content indexing in the new National Childhood and Adolescence Documentation and Analysis Centre website finds its place in this context.

\subsubsection{Adaptations of the Thesaurus: the Facets}

In order to apply a controlled vocabulary such as the ThIA to the web, it was necessary to make some modifications for more streamlined and user-friendly navigation, and also to gear it to unskilled users. The changes involved both structural and terminological levels, including facet clarification and a reduction of the levels in the hierarchy. In the web version of the vocabulary, the seven thematic areas have been removed, and the facets have been brought to the fore. As a matter of fact, the clarification of the facet structure allows for better navigation as a whole through:

- combining the focus of the facets (in the same way as with tag structure, to which many users are used); and

- the correlation of similar resources with a focus in common (so that the detailed file for a resource in the website suggests other semantically related resources).

In the new website, Minors, three main facets have been singled out, and that allow the description of individual documents according to specific characteristics:

1. Subject $=$ documents on the basis of their content;

2. Kind of resource $=$ documents on the basis of the kind of publication; and

3. Titles $=$ documents on the basis of their own titles or the titles of the collections.

Each facet represents an independent vocabulary for the user that nevertheless shares terminology and basic structure with the ThIA, apart from the third facet, which includes the title of the document described or of the collection.

The Subject facet is the core of the system. All of its terms come from the childhood and adolescence field 
and have been gathered together in 15 foci (Adoption, Foster care and reception services; Education, Learning and Educational services, etc.), which refer to the logical categories used in the thesaurus (activities, organisations, processes, etc.)

The focus tags can group together terms that are separated in the thesaurus, and they have been conceived to be highly meaningful for users in order to offer them a quick correlation to the topic, identifiable even before starting navigation among the subordinate terms.

The Kind of resource facet describes the document according to general categories that indicate:

- the kind of content (for example, Norms and sentences)

- the aim or function (for example, Bibliography, Meetings or study materials, etc.)

This facet includes terms which, in the ThIA, originate in the Culture thematic field, Cultural Tools facet, and Knowledge Organisation Tools and Representation Tools sub-facets. The Titles facet includes the titles of documentation produced by the centre and any related collections.

Within the facets, there is a moderate use of thesaurus relations; a basic hierarchical structure at the first level is visible only in the Subject facet, where, for some terms, explanatory notes are planned (not displayed at the moment), so that, for the users, their meaning and indexing use is always clear. In the future, it could be interesting to develop a system able to exploit the potential of the associative relationships, which, by reciprocal linking of terms that have very strict interchangeable and meaning superposition relationships among them, seems to be closest to free tagging, but without risking an uncontrolled expansion of the set of search terms, which was devised on the basis of accurate criteria.

\subsubsection{Adaptations of the Thesaurus: Terminology}

In traditional tagging on the web, one can accept terms that could not be used in a specialized controlled vocabulary, where the formal and semantic structure is more rigorous.

Therefore, in order to use the thesaurus for the website, it was necessary to modify the terms on a formal level so that they would become closer to users' natural language without losing their consistency and homogeneity.

For example, the term Minors out of the Family that we find in the Subject facet is not in the ThIA. By this term, we mean those minors (children, preadolescents, and adolescents) that are temporarily hosted either in other families (foster care families) or in centres for minors. Therefore, the term represents a set of concepts that in the thesaurus are indicated through accurate compound terms, each of them explaining a concept. If they are put within a rich and well-constructed network of thesaurus relations, these terms become clear also for the users and guarantee a high level of accuracy and precision in the answers to the query. On the other hand, their use in web searching would make the vocabulary cumbersome in lengthening the search keys, considered inconvenient in the web field.

It has been necessary to think accurately about the use of compound expressions, as it is a strong element of discontinuity with the thesaurus and its rules. For example, expressions such as Families and Family Relations and Childhood and Adolescence Social Policies have been created by combining terms that are separate in the thesaurus, and the possibility of permitting them is tightly linked to the indexing language chosen for the semantic description of documents in the library science field and to the need to use them in a pre-coordinated way (Gnoli 2004). As a matter of fact, the ThIA was developed for a pre-coordinated analytical-synthetic indexing system, where the terms are linked by the indexer according to precise syntactic rules in order to form complete and coextensive subject strings; a string of verbal terms is arranged in syntactic order using a system of role operators. These operators set each term in the context of the other terms in the string (AIB 1996). So the user, during his web search, starting from any term in the thesaurus, can go back to the subject strings that contain it and then to the documents linked to them. This procedure allows the expression and retrieval of even the most complex concepts through the combination of several terms from the thesaurus while respecting, at the same time, the constraints imposed by the string syntax. But this kind of system presupposes the use of pre-coordination, a highly useful method for catalogue searching, but less effective in the digital field, where better results are obtained by the post-coordination of terms, done by users at the moment of the query. On the other hand, a faceted structure (such as the one described here) works in the same way as post-coordinated indexing. As a matter of fact, users can start their search from any of the facets, selecting in each of them the most suitable focuses, so that the answer to their queries will be automatically created by the system regardless of their positioning. Therefore, in order to guarantee semantic retrieval of the documents described, it was necessary to create search keys using compound expressions.

\subsubsection{Indexing Criteria}

The system described respects the basic principles of subject indexing, both in the phase of conceptual analysis of the documents (standard ISO 5963) and in the organi- 
sation of the concepts; the use of these standards permits the expression of an accurate and coextensive subject statement and a precise categorization of the concepts, useful for their consistent attribution within the individual facets. Moreover, the system seeks to combine the semantic and syntactic structures as much as possible.

As a matter of fact, while it is true that, during their searches, users can select the facet from which their query can start together with the focus combination inside the facets, the answer is not displayed in a random way; on the contrary, it follows a precise citation order, based on the role that the concepts described have within the statement. That is, in the same way as for the subject strings created in a pre-coordinated field, the facets are arranged according to logical-linguistic standards, guaranteeing their readability and consistency. The first position is always taken by Subject as key concept (nuclear element); then, as extra-nuclear elements, come Kind of resource and Titles. These facets represent formal concepts, and they are linked by an instance relationship (common name/instance as expressed by a proper name, such as the title of a publication) where the Kind of resource facet represents the "non specific category" of the Titles facet.

Example:

\section{Subject: Unaccompanied Foreign Minors \\ Resource: Comments and analyses \\ Titles: Rassegne giuridiche}

\subsection{Cross-channel user journey}

While working on the new design of the National Childhood and Adolescence Documentation and Analysis Centre website and noticing the close interrelation of this area with the other documentation spheres of the Istituto degli Innocenti, Firenze (the museum, the archive, and the library) the idea came to link them together. More precisely, the idea is to create a series of informative paths that cross the different channels, that is to say that they traverse from the web to physical space and vice versa. In the end, there was both the opportunity and the need to plan a pervasive and cross-channel information architecture (Resmini and Rosati 2011) able to link together the different activity areas of the Istituto degli Innocenti, Firenze.

The basic idea is that the different activities of the Istituto degli Innocenti, Firenze function as contact points for the same service, so that the user's journey can start from any of the different points, but, at the same time, it can go on in a consistent and fluid way through to any other contact point - through the website, through the library, through the museum, or vice versa, without a break, from the point of view of the enrichment, extension, and variety of the visiting experience.

This plan is supported by:

- the idea of user journey, that is to say the strategic value of the paths conceived in a cross-channel key (Blandford and Attfield 2010);

- the thesaurus as bonding agent in this pervasive architecture, seeing the transversal applicability of the thesaurus to all the channels of the Istituto degli Innocenti, Firenze (those-such as the museum-that don't use the thesaurus in a systematic way could also easily make use of it). As it is a structure made of keywords (or tags - to use the web 2.0 terminology), the thesaurus allows tagging not only of web resources, but also of archival documents (or groups of documents), museum objects or paths, and library materials and services, establishing the possibility of passing from one to the other or-still remaining within the same channel—changing course ("berrypicking," see Bates 1989) through the hierarchical or associative relationships of a term with nearby ones. RFId, QR codes, and mobile devices allow these paths to be easily created in the physical world as well and to get them onto their digital counterparts.

\subsubsection{The Museum and the Archive}

The need to create a connection between these different channels was particularly relevant due to the creation of the new museum (Xi). The museum, apart from collecting the works of artists that have made this reception space beautiful and unique, aims to create virtual online exhibitions and thematic paths in order to tell the history of the Istituto degli Innocenti, Firenze and of its activities, linking together documents of different natures and origins (from archival documents to the most recent contributions linked to new documentary activities).

To support the user during the visit, next to traditional analogue equipment (banners, texts, audio-visuals), the new museum plans to use RFId systems and portable devices with net connections, through which integrative digital content can be viewed, and, at the same time, it will be possible to personalise the visit path. Highly significant for the realisation of these projects are the sources coming from the historical archive. The relevance of its heritage lies in the uniqueness of the pieces and in the consideration that many archive series have a chronological continuity that allows significant research over long periods of time. At the moment, consultation of the archive is possible through its online inventory, which allows users to navigate among funds, sections, sectors, and information cards, making them understand the complex- 
ity of the hierarchical structure thanks also to the graphical display of the results. The semantic indexes which will allow subject access have not been created yet. In the next paragraph, we will see that some archival materials have been indexed in an experimental way with descriptors coming from ThIA with the prospect of integration between different information channels, as described so far, or to offer to archive users also the possibility of content access.

\subsubsection{The Children of Italy Exbibition and the Digital Repository}

The experiment described here, reported at the workshop ISKO in Rome, October 2012, was inspired by the exhibition Children of Italy: Istituto degli Innocenti, Firenze and the birth of a childhood national project (1861-1911), open from December 3, 2011 to March 18, 2012, and then extended until June 3, 2012. The exhibition traces the history of the Istituto degli Innocenti, Firenze and the development of national childhood policies in the first 50 years following Italian Unification. Through the biographies of some of the children who lived in the Istituto degli Innocenti, Firenze and in other Italian charitable institutions between 1861 and 1911, old pictures, objects, videos, and archival documents, the daily life of children inside the institutes is recounted; the evolution of their reception, their care, and education, the demographic aspects and the life paths that awaited them in the new nation, the care for pregnant women. Information-rich legends support and accompany the archive documents and the 40 photos on display, coming from the historical Archive of the Istituto degli Innocenti, Firenze and from other important collections. A report of this event was published on the centre's website and gave rise to the opportunity to analyse the topic of child abandonment and care not only from a historical perspective, but also with a look at the condition of children in contemporary society.

The experimentation leading to a cross-channel thematic path starts from here, from the desire to give the web user the chance of finding an organised and navigable knowledge space on the topics discussed in the article. The stages that led to the realisation of this path were:

- The cataloguing by subject of the initial document according to traditional indexing (conceptual analysis, formulation of the subject statement and translating it into facets). According to the analysis, the document shows itself to be a news report that analyses the topics of child abandonment, the condition of children outside of the family, and reception services. Apart from these main concepts, it is possible to identify other topics that, even though they are not principal, have definite relevance to the main topic (such as, for example, family foster care; the history of Istituto degli Innocenti, Firenze; the daily life of children inside institutes; and so on). The main topics are expressed through the Subject, Kind of Resource, and Titles facets as shown above.

Navigating the facets will allow the users to conduct their searches inside the site, widening or limiting according to well-thought-out, non-random procedures.

- The creation of a network of contextual links based on the subject statement (main subjects and secondary subjects). These links will lead the user out of the site, putting him in contact with different information channels, where his search can be deepened or reoriented: for example, bibliographies or filmographies in the centre catalogue (http://opac.minori.it/EOSWeb/ OPAC/Index.asp), thematic visual reference desk, or digitized documents from the museum or archive. In relation to the topic discussed, these last materials are of particular interest. These materials are managed through the Digital Repository, a preservation tool for digitized materials developed by a group of Italian companies specialising in the field of digitalization and kindly offered so that this application could be tested.

Inside the repository were put some museum objects and archival documents, which were appropriately digitized and linked to the topics dealt with in the news report from which we started:

- a digitized reproduction of a painting (the Madonna degli Innocenti);

- a picture (the reinforced window of the Istituto degli Innocenti, Firenze); and

- some archival documents (the history of Demetrio), etc.

Inside the repository, the physical characteristics of the materials were described and they were indexed using the thesaurus terms. The user can enter the repository from the web by starting from one of the terms used in the Subject facet to describe the news report, and he can find, in this way, a series of documents with similar content; once inside the repository, it is possible to navigate via the thesaurus terms to redefine and refine the search.

\subsection{Conclusions}

In this paper, we have seen how different channels and contexts (different in their nature, functions and interface) could be related one to another to create a unique 
ecosystem, exploiting a common information architecture, so as to allow a continuity of experience among various contexts and a systematic integration of the institute's activities and faces. The basis for this process is a product of the documentation field: the thesaurus. It can become the tool that can help further an overall knowledge of the actions and of the topics concerning childhood and adolescence, either from a historical perspective, or as a contemporary life condition.

Using the thesaurus tools, moreover, it will be possible to move from the digital channels of the Istituto degli Innocenti, Firenze (the website, the online catalogue, the digital repository) to the physical ones (such as the library, the museum or the archive), but not only in a generic way, on the contrary with specific subjects as a compasssubjects expressed through the ThIA terms or those modified for the web. In this way, it will be possible to pursue the visit experience by choosing specific paths within the physical space, or, vice versa, it will be possible to move from the real to the digital world. More than that, through mobile devices, it will be possible to develop an integrated use of web resources during a visit in physical space, in order to personalise the path, and find objects or related information.

The next step after the single channel correlation is the cross-channel one, between physical and digital space.

\section{References}

AIB. GRIS. 1996. Guida allindicizzazione per soggetto. Roma: AIB.

Bates, Marcia J. 1989. The design of browsing and berrypicking techniques for the online search interface.
Online review 5: 407-24. Available http://www.gseis. ucla.edu/faculty/bates/berrypicking.html.

Bianchini, Carlo. 2011. Organizzare la conoscenza con la sequenza di filiazione della

Bianchini, Carlo. 2012. Colon Classification and nuovo soggettario: the case of the Library of the Natural History Museum of Udine, Italy. Knowledge organization 39: 23-28.

Blandford, Ann and Attfield, Simon. 2010. Interacting with information. Available http://www.morganclaypool.com/ doi/pdf/10.2200/S00227ED1V01Y200911HCI006

Centro nazionale di documentazione e analisi per l'infanzia e l'adolescenza. 2007. Thesaurus italiano infanz̧ia e adolescenza. Firenze: Istituto degli Innocenti. Available http://www.minori.it/thesaurus. And reference (ThIA 2007) replaced for (Centro nazionale di documentazione e analisi per l'infanzia e l'adolescenza 2007).

Giannetto, Marina. 2011. Mostre virtuali online. Linee guida per la realizzazione: la genesi di un progetto per il web culturale. DigItalia 6n1: 147-60. Available http:// digitalia.sbn.it/riviste/index.php/digitalia/article/view/ 498/346.

Gnoli, Claudio. 2004. La classificazione a faccette. Roma: AIB.

Resmini, Andrea and Rosati, Luca. 2011. Pervasive information architecture: designing cross-channel user experiences. Burlington: Morgan Kaufmann.

Rosati, Luca. 2003. Per un accesso multidimensionale allinformazione, o della classificazione a faccette. Available http:// iainstitute.org/it/articoli/000204.html.

Rosati, Luca. 2011. Classificazione. Available http://luca rosati.it/guide/classificazione. 